\title{
Inhalt vom Band 259
}

\author{
Heft 1
}

Ausgeseben im Màrz 1978

-Wer und H. LaNDe: Untersuchung von Strömungsvorgängen beim Stoffübergang in bzw. aus düranen Flüssigkeitsfilmen. Mit 7 Abb. (Eing. 20. 7. 76)

Sümel, K. Gustav, R. Paktzold und J. Fabian: Spektroskopie und Photochemie indigoider Verbindungen. II. Theoretische Untersuchungen am Grundchromophor des trans- und cis-

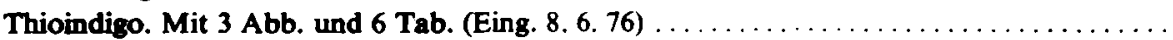

1. GumanN, I. SCHOAER und D. HABgRLAND: Thermokinetische Untersuchung der Reaktion von Pentacyanokobaltat(II) mit organischen Halogenverbindungen. I. Thermokinetische Methoden unter anaeroben Arbeitsbedingungen, Meßwerterfassung und kinetische Auswertung. Mit 2 Abb. und 1 Tab. (Eing. 13. 8. 76)

Danmelewicz-Ferchmin: Hydrogen Bonding and Anomalous Saturation. With 5 Fig. (Received 22. 7. 76).

. Stbesser, M. Segonund und B. Pritze: EPR-Untersuchungen an polykristallinen und matrixstabilisierten EDA-Komplexen des TCNQ und TCNE. Mit 5 Abb. und 2 Tab. (Eing. 3.9. 76) . .

HAM MANG TAN: Elektrochemisches Verhaiten ionenimplanticrter Si-Elektroden in flußsaurer Lösung. Mit 5 Abb. (Eing. 16. 9. 76)

A. Kirllov, J. K. DelmarsKiJ und W. Horlbeck : Die Temperaturabhängigkeit der Linien in den Raman-Spektren des kristallinen Natriumrhodanids und der Umorientierungsmechanismus des Rhodanidions. Mit 2 Abb. und 2 Tab. (Eing. 11. 8. 76)

-P. Pisissner, H. Lucius und G. Smettan: Erfassung und Verarbeitung von Meßdaten einer stopped-flow- und Temperatur-Sprung-Apparatur mit dem Kleinrechnersystem KRS $\mathbf{4 2 0 0 .}$

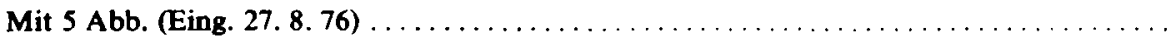
ILLGEN und J. SCHEVE: Untersuchung der Wechselwirkung von $\mathrm{O}_{2}$ und $\mathrm{CO}$ mit Kobalt- und Nickelferrit. I. Untersuchungen der elektrischen Leitfähigkeit bei der Wechselwirkung von Kobalt- und Nickelferrit mit den Reaktionspartnern. Mit 4 Abb. und 3 Tab. (Eing. 2.9. 76)... MiCHEL, E. ANGEIÉ und W. MeILER : ${ }^{13} \mathrm{C}$-NMR-Untersuchungen von Silber-Olefin-Komplexen in Lösung. Mit 2 Tab. (Eing. 3. 8. 76)

Michel: Zur Berechnung der freien Enthalpie innerer Spannungsfelder bei Entmischungs-

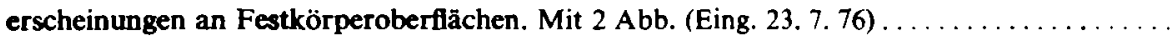
Strange: Zur Bestimmung der Dicke immobiler Wasserschichten auf $\mathrm{SiO}_{2}$-Oberflächen mit Hilfe der relativen Viskosităt verdünnter Suspensionen. Mit 3 Tab. (Eing. 10. 10. 75, in rev.

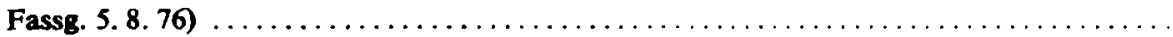
LAszí und G. BaktaI: Zur partiellen Stofftrennung bei der Wechselstromelektroosmose. III. Mit 2 Abb. und 2 Tab. (Eing. 4. 10. 76).

Finster, P. Lorenz und E. Angele: Photoelektronenspektroskopische Untersuchungen an AgNaA-bzw. AgCaNaA-Zeolithen. Mit 2 Abb. und 6 Tab. (Eing. 31. 8. 76)

MÜLLER und CH. OPITz: Eigenschaften kleiner Teilchen. Dissoziative Chemisorption und Wechselwirkungen chemisorbierter Atome an ein- und zweidimensionalen Metallkristalliten. Mit 4 Abb. und 1 Tab. (Eing. 30. 6. 76, in rev. Fassg. 17. 9. 76) $\ldots \ldots \ldots \ldots \ldots \ldots \ldots \ldots$ . A. Lawrenko, W. L. Tikusch, W. S. Senkow, W. A. Kramez, K. W. Nasarenko und W. M. Werestschak: Die Adsorption von molekularem und atomarem Wasserstoff an den Flächen von $\mathrm{MgO}, \alpha-\mathrm{Al}_{2} \mathrm{O}_{3}$ - und $x$-SiO $\mathrm{Sin}_{2}$-Einkristallen. $\mathrm{Mit} 6$ Abb. und I Tab. (Eing. 8. 6. 76) ..... 
P. Volatie, M. Giurgea and D. Sandllescu: Excess Thermodynamic Quantities of Aromatic Hydrocarbon Solutions in Polar Solvents, Determined by Rayleigh Light Scattering. With

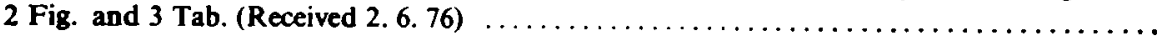

H. ScHmm: Die Thermodynamik der Zwischenreaktionen der Verdampfung des Wassers. Mit 1 Abb. und 3 Tab. (Eing. 5. 8. 76)

R. Salzer, J. Fruwert und G. Franz: Konformationsuntersuchungen an aliphatischen Alkoholen. Mit 2 Abb. und 1 Tab. (Eing. 24. 8. 76).

Kurze Mitteilungen

O. E. S. Godinho and E. SteIN: The Stability Constant of Cobalt(II) Monoazide Complex. With 1 Tab. (Received 15.11.76)

R. V. Gopala RaO, R. Joardar and T. Nammal.var: On the Resistivity of Liquid Metals. With 2 Tab. (Received 11. 4. 77)

H. Kasperskı, J. Hellebrand, H.-K. Roth und M. RäTZSCH: UV-initiierte Radikalbildung bei Maleinsäureanhydrid gelöst in Tetrahydrofuran und 1,4-Dioxan. Mit 2 Abb. (Eing. 28. 2. 77). .

J. BeNDIG und D. KREYSIG: Lösung der Fluoreszenz des Akridiziniumions durch schweratomhaltige Donatoren. Mit 2 Abb. und I Tab. (Eing. 21. 3. 77)

H.-J. BrTTRICH und G. Schmark: Dielektrische Untersuchungen zur Elektronen-Donator-Akzeptor-Wechselwirkung in Mischungen. Mit $2 \mathrm{Abb}$. und $2 \mathrm{Tab}$. (Eing. 20.11. 76, in rev. Fassg. 11. 5. 77).

Buchbesprechungen

\section{Heft 2}

Ausgegeben im Maj 1978

I. A. Ammar, S. Darwish and M. Khalll: Cathodic Hydrogen Evolution on Bismuth in Acid Media. With 9 Fig. and 2 Tab. (Received 21.3.75)

I. A. Ammar, S. Darwish and M. Selim: Effect of Thiourea on the Acid Corrosion and Electrochemical Polarization of Nickel. With 11 Fig. and 5 Tab. (Received 21. 3. 75)

K. Kumari, S. Prakash and B. Krishna: Kinetics and Mechanism of the Reduction of cis- and trans-diaquotetramminecobalt(III) by Cysteine. With 2 Fig. and 2 Tab. (Received 26.7.76)...

D. Freude, U. LohSE, D. Michel, H. Pfeifer und H.-J. ZäHR: Magnetische Kernresonanzuntersuchungen zum Protonenaustausch zwischen Hydroxylgruppen in Zeolithen und adsorbierten Wasser- bzw. Buten-1-Molekülen. Mit 6 Abb. und 3 Tab. (Eing. 12. 10. 76)...........

H. S. RANA and J. P. TANDON: Mixed Ligand Complexes of Some Rare Earths. La(III), Pr(III) of Nd(III) HEDTA-Hydroxy Acids. With 4 Fig. and 1 Tab. (Received 12. 5. 76) ...........

M. S. Dhullon: Solid + Liquid Phase Diagrams of Mixtures Containing Phenol, Cresols and Dihydroxyphenols. With $5 \mathrm{Fig}$. and I Tab. (Received 12.8. 76) $\ldots \ldots \ldots \ldots \ldots \ldots \ldots \ldots$

M. S. Dhillon and B. S. MAhl: Surface Tensions of Mixture of Chlorobenzene with Cyclohexane, Benzene, o-, m- and p-Xylenes. With 5 Fig. and 1 Tab. (Received 12. 8. 76) .....

O. Grossmann: Bestimmung der Solvatationszahlen bei der Extraktion von assoziierten und polymerisierten Teilchen. Mit 1 Abb. und 2 Tab. (Eing. 19. 4. 76) $\ldots \ldots \ldots \ldots \ldots \ldots \ldots \ldots$

A. KuBrTz: Kernspin-Gitter-Relaxationszeituntersuchungen in den flüssig-kristallinen Phasen von Oktyloxy-benzoesäure-4-nitrophenylester. Mit 4 Abb. und 1 Tab. (Eing. 3. 11. 76) ........

P. Frölıch und F. Wolf: Ein neues Kriterium zur Prognose des kinetischen Mochanismus von Ionenaustauschreaktionen. Mit $2 \mathrm{Abb}$. und 5 Tab. (Eing. 5. 11. 76) 
R. STósere, J. SAUER, U. EwerT, J. FrTEDRICH und R. IücK: EPR- und UV-spektroskopische Untersuchungen zur Elektronenstruktur von $\mathrm{CU}(\mathrm{II})$ - und VO(Il)-Phenacylpyridin-Komplexen.

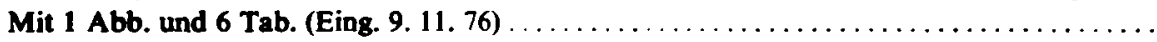

A. Szymax́sxu, A. Huczro and A. Podgórski: Synthesis of Silicon Nitrides in a Low-Temperature Plasma Jet. With 2 Fig. and 2 Tab. (Received 30. 9. 76) $\ldots \ldots \ldots \ldots \ldots \ldots \ldots \ldots \ldots$

D. Veselinović, F. BAJRakTaRr and D. Marković: Complex Compounds of Vanadium and 1,4Dihydroxybenzene in Sulphuric Acid Solutions. With 3 Fig. and 1 Tab. (Received 7. 10. 76) ...

H. YamaoKA: Comment on the Earlier Literature on the Complex Ion Formation Constant of Hexaammine- and Tris-Ethylene-diamine-Cobalt(III) Ions. (Received 11. 10. 76)

G. F. Cerofol.nNI: Heterogeneity Effects in Chemisorption Kinetics. With 1 Fig. (Received

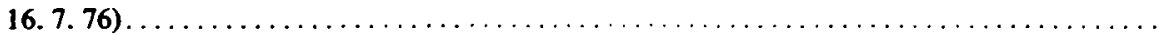

G. F. Cerofolin: Adsorption on Homogeneous Surfaces. A Statisticomechanical Way of Accounting Collisions which Lower the Rate of Desorption. (Received 4. 11. 76) ...........

N. TAKEUCHI, K. INABE and J. Yamastra : Valence Conversion Kinetics of Impurity Ions in MgO Single Crystals by UV Irradiation. With 2 Fig. and 1 Tab. (Received 11.10 .76 ) ........

M. Solc: Mean Lifetime of Energized Molecules. With 1 Tab. (Received 4. 11. 76) .........

L. MülleR und H. MORTrz: Der Einfluß einer Eisendeuteroporphyrinmonoschicht auf die Reduktion von Sauerstoff an Pyrographitelektroden. Mit 2 Abb. (Eing. 20. 10. 76) .............

R. WetzeL, L. Müllar und E. KrauSE: Der Einflul3 von Ko-Adsorbatschichten auf die elektrokatalytische Oxydation einiger organischer Brennstoffe an Pt-Elektroden. Mit 8 Abb. (Eing. 20. 10. 76) . . . . . . . . . . . . . . . . . . . . . . . . . . . . . . . . . . . .

\section{Kurze Mitteilungen}

R. SiEgemund: Quantenchemische Rerechnungen an Modellen von Elektrodengrenzflächen.

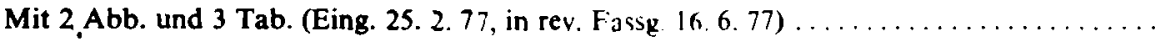

E. Bich, J. Millat, G. OPEL und E. VoGf L: Zur zwischenmolekularen Wechselwirkung im Dampf von Fluorbenzol. Mil 3 Tab. (Eing. 25. 5. 77)

G. Figurskı und M. Mühnenbruch: Das Dampf-Flüsilgkeits-Gleichgewicht des Systems Aceton(1)/Vinylacetat(2) bei 760 Torr. Mit 3 Abb. und 4 Tab. (Eing. 27. 5. 77) . . . . . . . . .

W. Kühnel, Th. Steiger, E. Gey und H.-J. SPANGenber(;: Quadrantenchemische Berechnung der Bildungswãrmen ausgewählter $\mathrm{C} / \mathrm{H}$-haltiger Spezies mit der MINDO-Methode. Mit 2 Tab.

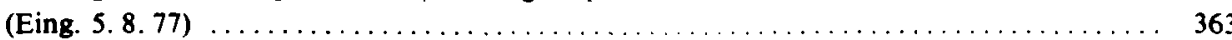

R. FeISTEL: A New Result for the $c \log c$-Term of Electrolytic Conductance. (Received 4. 6. 77) ..

H. Kehlen und E. FuhrmanN: Die Exzeßenthalpien in Butylacetat-Butanol-Mischungen. Mit

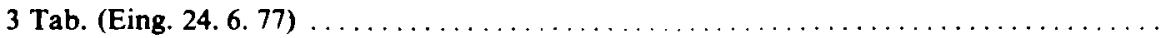

W. RudziŃski, A. Patrykiejew and J. Narkiewicz : Critical Properties of Bergmann's Model for Mobile Monolayer Adsorption. (Received 12.3. 77. in re, version 30. 6. 77) . . . . . . . . 377

H. KEHLEN: Zur Berechnung partieller molarer Größen aus molaren Größen. (Eing. 29. 8. 77) 380

\section{Heft 3}

Ausgegeben im Juni 1978

D. KUNATH und K. MöLlER: Infrarotspektroskopische Lntersuchungen zur Adsorption von Ammoniak an A-Zeolithen. Mit $8 \mathrm{Abb}$. und $3 \mathrm{Tab}$.(Eing. 22. 6. 76, in rev. Fassg. 25. 10. 76) ...

G. GrBHARDT: Theoretische Betrachtungen zur Frontalch romatographie an Ionenaustauschersäulen. 1. Mitt. Mit 6 Abb. und 1 Tab. (Eing. 16. 11. :61 
G. GeBhARDT: Theoretische Betrachtungen zur Frontalchromatographie an Ionenaustauschersäulen. 2. Mitt. Mit 3 Abb. und 3 Tab. (Eing. 16. 11. 76) $\ldots \ldots \ldots \ldots \ldots \ldots \ldots \ldots \ldots$

D. JanNakoudakis und E. Theodoridou: Spektrophotometrische Untersuchung der Bildung von Ionenpaaren durch Protonenübergang zwischen den Nitronaphtholen und den Piperidin in verschiedenen Lösungsmittein. Mit 2 Abb. und 5 Tab. (Eing. 11.11.76) $\ldots \ldots \ldots \ldots \ldots \ldots$

D. Freude, H.-J. Herden, H. Pfeifer, H. Schmiedel und R. Schöllner: Magnetische ${ }^{\top}$ Li-Resonanzuntersuchungen zur Anordnung und Beweglichkeit der Lithiumionen in dehydratisierten A-Zeolithen. Mit 2 Abb. und 1 Tab. (Eing. 25. 11. 76) .

R. B. Subramanian and B. P. Sinha: Kinetics and Mechanism of the Oxidation of Thallous lon by Ceric Ion. Part VII. With 1 Fig. and 6 Tab. (Received 11.11. 76)

D. Hartmann, G. Herzog und K. SChelmann: Luminophore auf der Basis aktivierter ZinkYttrium-Mischoxide für die Anwendung in Cadmiumdampf-Niederdruckentladungslampen. I. Emission und Anregung mono-, bi- und triaktivierter Zink-Yttrium-Mischoxide mit aquimolaren Mengen beider Grundgitterkomponenten. Mit 8 Abb. (Eing. 6. 12. 76) ..........

J. SChmelzer und K. Quitzsch: Phasengleichgewichtsuntersuchungen an Mehrkomponentensystemen. II. Isobares Flüssigkeit-Dampf-Gleichgewicht und Flüssigkeit-Flüssigkeit-Gleichgewicht in den ternären Systemen Methyläthylketon-Wasser-Diisobuten, Methyläthylketonsek. Butanol-Wasser und sek. Butanol-Wasser-Diisobuten. Mit 7 Abb. und 2 Tab. (Eing. 11. 8. 76, in rev. Fassg. 7.12. 76)

W. NAumanN und W. STILleR: Strahlenchemische Radikal-Reaktionen beim Ubergang von der inhomogenen zur homogenen Verteilung der Reaktanten. Mit 2 Abb. (Eing. 8. 12. 76) .....

U. DECKER, R. HAHN und G. RuDAKOFI : Ionensolvatation und Ionenassoziation in elektrolythaltigen Lösungsmittelgemischen. Mit 11 Abb. (Eing. 24. 5. 76, in rev. Fassg. 18. 12. 76) . .....

M. MORITZ, M. HintZe und L. Müller: Zur elektrochemischen Reduktion des Kupfer(I)trans(14)-dienperchlorats in Pufferlösungen an einer Graphitelektrode. Mit $8 \mathrm{Abb}$. (Eing.

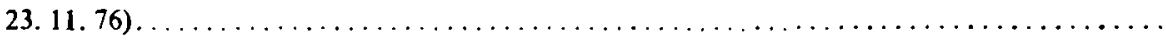

H.-G. FrıTSChE: Approximative Lösungen der SW-X $\mathrm{X}_{a}$-Methode für Cluster. I. Genähertes Atompotential, muffin-tin-Näherung und Rumpfzustände. Mit 4 Abb. und 1 Tab. (Eing. 6. 12. 76) ...

H.-J. SPANGEnBerg, I. BÖRger, H. HoffmanN und G. MÖGEL: Zur Bildung höherer Acetylenderivate bei der Plasmapyrolyse von Kohlenwasserstoffen und der Rolle des $\mathrm{C}_{2} \mathrm{H}$-Radikals. Mit

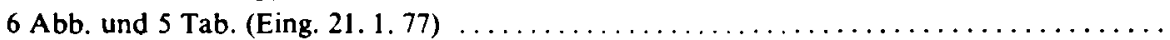

W. HoERSTEL: Zur elektrischen Wirksamkeit von Jod im Halbleiter Tellur. Mit 2 Abb. und 1 Tab. (Eing. 17. 11. 76)

J. BeNDIG und D. KREYSIG: Desaktivierungsverhalten von Arenen und Heteroarenen. III. Die Berücksichtigung der Reemission bei der Fluoreszenzlöschung. Mit 2 Abb. (Eing. 21. 12. 76) ...

J. HeymanN, L.-G. Fleischer, G. S. Jablonsky und H. G. Slinko: Lineare und nichtlineare thermodynamische Materialgleichungen (constitutive equations) chemischer Reaktionen und ihre variationstechnische Ableitung. Mit 1 Abb. (Eing. 1.6. 76, in rev. Fassg. 16. 12. 76) ...

\section{Kurze Mitteilungen}

K.-H. Radeke, P. Struve und K. Ehrhardt: Auswertung adsorptionskinetischer Versuche bei konstantem Volumen und variablem Druck. Mit 3 Tab. (Eing. 11. 7. 77, in rev. Fassg. 27.9.77)

R. RADEGLIA : Zur Berechnung individueller thermodynamischer Aktivitätskoeffizienten einzelner Ionensorten in Elektrolytlösungen nach A. Ferse. (Eing. 26. 6. 77, in rev. Fassg. 14. 7. 77) . .

A. FrRSE: Erwiderung auf die Bemerkung von R. Radeglia zum Vorschlag zur Berechnung indivi-

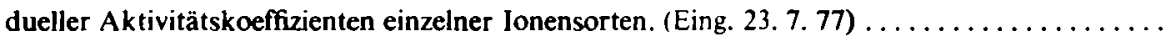

R. RADEglia : Anmerkung zur voranstehenden ,Erwiderung“. (Eing. 20. 8. 77) ............

D. Gelarn, K.-P. Roethe, G. SchöN und P. Struve: Über Porendiffusion und Barrierenwider-

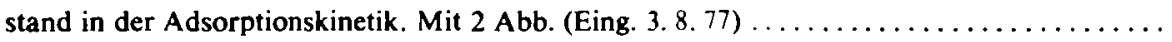

M. L. Sood and G. KaUR: Diffusion Coefficients of Potassium Fluoride Water System from Diaphragm Cell Experiments. With 4 Fig. and 3 Tab. (Received 17. 3. 77) .............. 


\section{Heft 4}

Ausgopeben im August 1978

G. GEBHARDT: Theoretische Betrachtungen zur Elutions-Chromatographie an Ionenaustauschersảulen. 4. Mitt. Min 5 Abb. und 3 Tab. (Eing. 16. 11. 76) .

E. KALMÁN und G. PAlINkÁs: Die Beugungsexperimente und die Theorie der Elektrolytlösungen.

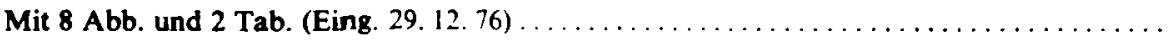

H. Seitel, C. Bayer, J. Fruwert und G. Geiseler : Simultane OH...O- und OH...t $t$-Wasserstoffbrücken in Phenol-Anisol-Komplexen. Mit $4 \mathrm{Abb}$, und 3 Tab. (Eing. 11. 8. 76, in rev. Fassg. 5. 11. 76).

J. Maranon and O. M. Sorarrain: Intramolecular Charge Transfer for Some Singlet and Triplet States in the Molecules of Diacetamide and N-methyldiacetamide. With 1 Tab.

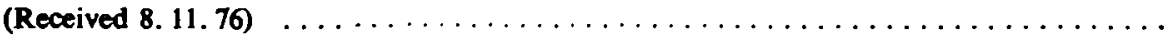

T. Szalay, T. Német und L. Bartha: Untersuchung von Grenzflächenvorgängen an WolframOxiden. III. Uber in wäBrigem Medium an Wolframoxid nichtstöchiometrischer Zusammensetzung $\left(\mathrm{WO}_{2,87}\right)$ stattindenden Ionenaustausch-Reaktionen. Mit $6 \mathrm{Abb}$. und $1 \mathrm{Tab}$. (Eing. 15. 11. 76).

B. SwAROOP: Thermodynamic Studies on Solubilized Solutions of Drugs with Special Reference to

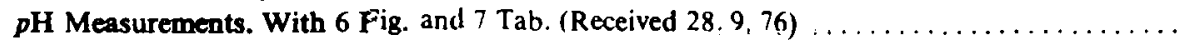

U. Mrssow, U. DoYt und D. KuCHENkECKER: Thermodynamische Untersuchungen an Lösungsmittel/n-Parafin-Systemen. IX. Die Systeme Butanon(1)/n-Dodecan(2), Butanon(1)/n-Hexadecan(2) und Butanon(1)/n-Octadecan(2). Mit 1 Abb. und 2 Tab. (Eing. 23. 11. 76, in rev. Fassg.

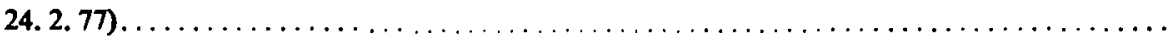

G. KNOBLOCH, E. ButrizR und S. ReIFFARTH: Die Anwendung des Krupkowski-Modells auf das

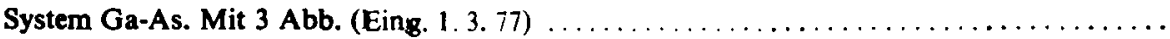

S. Soxolowskr: Statistical Mechanics of Adsorption Phenomena. Virial Expansion, Equation of

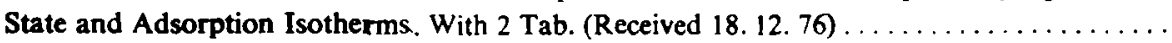

J. BENDIG, R. SCHÓNEICH und D. KREYSIG: Auswertung von UV-Vis-Spektren organischer $\pi-\pi$ Molekülkomplexe. XIV. ct-Anregungsenergien diarylsubstituierter Äthylene und Butadiene-

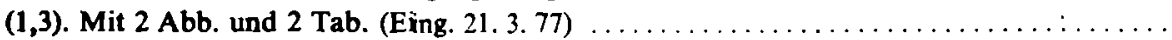

R. Mitzner, C.-R. Kramer, G. Kempter und D. Hellmann: Zur basischen Hydrolyse von Phenoxyessigsãurcestern. I. Zur Kinetik der basischen Hydrolyse einiger 2,4-Dichlorphenoxyessigsăurephenylestern und von 2-Methyl-4-chlorphenoxyessigsäure-4-methylphenylestern in $20 \mathrm{Vol}-\%$ igem Äthanol-Wasser-Gemisch. Mit $2 \mathrm{Abb}$. und 2 Tab. (Eing. 7. 2. 77) .........

R. MITZNER und C.-R. KRAMER: Zur basischen Hydrolyse von Phenoxyessigsäureestern. III. Zur basischen Hydrolyse von 2,4-Dichlorphenoxyessigsäurealkylestern. 1. Teil. Mit $1 \mathrm{Abb}$. und 6 Tab. (Eing. 23. 2.77)

O. Brede, J. Bós, W. Helmstrert und R. MehNeRT: Pulsradiolyse polymerisationsfăhiger Verbindungen. Energieübertragungsprozesse in System Benzol-aromatisches Olefin. Mit $8 \mathrm{Abb}$. und 1 Tab. (Eing. 1. 12. 76), in rev. Fassg. 20. 2. 77)

F. Wolf, K. Ggorgi und K. Prlchowski: Zum Ionenaustausch einwertiger Kationen am synthetischen Mordenit. I. Gleichgewichte und Thermodynamik des Austausches $\mathrm{K}^{+} / \mathrm{Na}^{+}, \mathrm{H}^{+} / \mathrm{Na}^{+}$ und $\mathrm{H}^{+} / \mathrm{K}^{+}$. Mit $7 \mathrm{Abb}$. und 1 Tab. (Eing. 17. 3.77)

L. MülLER: Zur Allgemeingültigkeit der linearen freien Enthalpiebeziehung für chemische Elementarreaktionen. (Eing. 21. 2.77). .

M. Bülow und U. Werner: Zur Berechnung von Aktivitätskoeffizienten für die Komponenten binărer Adsorbatgemische. Mit 1 Abb. (Eing. 8. 2. 77)

R. Mehinert, O. Brede, J. Bơs und W. HeLMSTRErT: Pulsradiolytische Untersuchung von Elementarreaktionen der strahleninitiiertten radikalischen Polymerisation des Styrols und 1-Methylstyrols. Mit 5 Abb. und 1 Tab. (Eing. 7. 10. 76, in rev, Fassg. 16. 3. 77) . . . . . . . . . . 
O. Herbarth und F. Plümer: Komplexe Untersuchungen zur strahlungsinitiierten Polymerisation. II. Pulsradiolytische und stationäre Leitfahigkeitsuntersuchungen zur radikalisch verlaufenden Strahlungspolymerisation von MMA und Styrol. Mit 5 Abb. und 1 Tab. (Eing. 3. 3. 77) ....

D. LOHSE, E. KüHRRR und K. SCHWETICK: UV-spektroskopische Bestimmung der Quantenausbeuten von Parallelreaktionen. Quantenausbeuten des Photoabbaus von N, N'-Diphenylharnstoff und der Photo-Fries-Reaktionsprodukte. Mit 3 Abb. und 3 Tab. (Eing. 22. 10. 76, in rev. Fassg. 24. 2. 77)

\section{Kurze Mitfeilungen}

H.-D. Dörfler und H. SACKmanN: Integrale Lösungsenthalpien von Na-n-oktyl- und Na-nnonylsulfat im mizellaren Konzentrationsgebiet. Mit 2 Abb. und 1 Tab. (Eing. 4. 8. 77) ....

H.-J. Polster, B. MülLeR-Gobrecht und H.-J. Bittrich: Ultraschallgeschwindigkeit und isotherme Kompressibilität in den Systemen Cyclohexan-N-Methyl- $\varepsilon$-caprolactam und n-HexanN-Methyl- - -caprolactam. Mit 1 Abb. und 3 Tab. (Eing. 18. 8. 77) $\ldots \ldots \ldots \ldots \ldots \ldots \ldots$

H. Kresse, J. Pietscher, H.-J. Deutscher, D. Demus und W. Welssflog: Dielektrische Relaxation kristallin-flüssiger 4-Methoxybenzoesāure-[4-n-alkyloxy-phenylester] im MHz-Bereich.

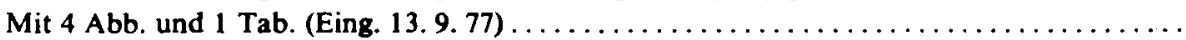

J. Kärger, H. Pfeifer, M. Rauschrr und A. Walter: Selbstdiffusionsverhalten der homologen Reihe der n-Alkane von n-Butan bis n-Oktadekan in NaX-Zeolithen. Mit 5 Abb. und 1 Tab. (Eing. 9. 12. 77)

H. Müller und Сh. OPITz: Zur Wechselwirkung chemisorbierter Molekūle. Mit 2 Abb. (Eing.

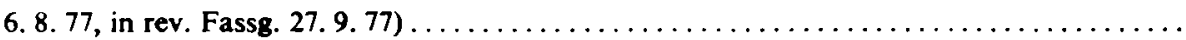

K. SAlzER: Berechnung der ${ }^{13} \mathrm{C}$-Kernresonanzverschiebung infolge von Adsorption für Butene

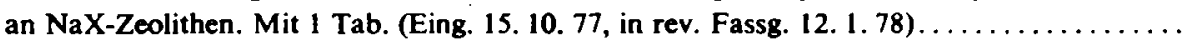

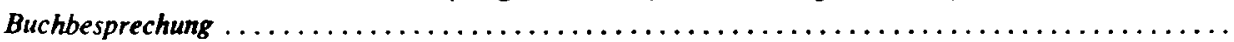

\section{Heft 5}

Auspogeben im Oktobor 1978

B. Singh, P. K. SAXENA, KM. M. Richards and B. Krlshna: Kinetics of Oxidation of Methyl Iso-Propyl Ketone by Ce(IV) in Sulphuric Acid Solution. With 1 Fig. and 2 Tab. (Received

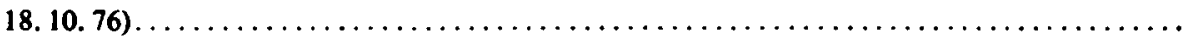

M. M. ZAFAR and G. N. Chaudry: The Effect of Stationary Hydrogen Chloride on Poly (Vinyl Chloride) Degradation in Nitrobenzene. With 1 Tab. (Received 12.4.76) ..............

M. M. ZAfar and G. N. Chaudry: Fractional Rate of Styrene Polymerisation in Solution. With

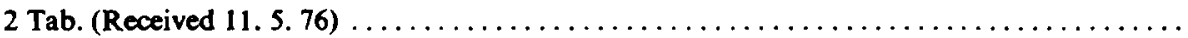

B. P. Sinha and R. B. Subramanian: Kinetics and Mechanism of the Oxidation of Thallous Ion by Ceric Ion. Part VIII. With 2 Fig. and 5 Tab. (Received 2. 12. 76) ..............

W. C. Vasudeva, A. Hossady, S. N. Srivastava and S. Wasif: A Kinetic Study of the Oxydation of Glyoxal by Peroxydisulphate. With 5 Fig. and 4 Tab. (Received 15.6.76, in rev. version 27. 1.77).

R. D. SINGH: CNDO/2 Study of NH...O Hydrogen Bond in Imidazole... - Glycine+ Complex. With 5 Fig. (Received 3.1.77)

CH. WOHLFARTH und M. T. RätzSCH: Untersuchungen zum 2. dielektrischen Virialkoeffizienten polarer Gase. 2. $\mathrm{C}_{2} \mathrm{H}_{3} \mathrm{Cl}, \mathrm{CHF}_{2} \mathrm{Cl}$ und $\mathrm{CHFCl}_{2}$. Mit 4 Abb. und $5 \mathrm{Tab}$. (Eing. 30. 11. 76,

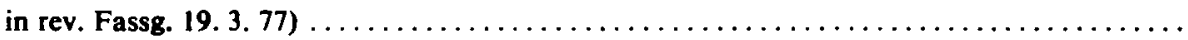

F. F. HANNA and A. M. BishaI: Dielectric Relaxation in Dilute Solutions of Some HydroxyCompounds. With 2 Fig. and 2 Tab. (Received 13. 1. 77)

F. Wol.F, K. Georgi und K. PIlchowski: Zum Ionenaustausch einwertiger Kationen am synthetischen Mordenit. II. Zur Kinetik des Austausches $\mathrm{H}^{+} / \mathrm{Na}^{+}$und $\mathrm{H}^{+} / \mathrm{K}^{+}$. Mit $3 \mathrm{Abb}$. und 1 Tab. (Eing. 17. 3. 77) 
R. Mniss und K. Lunkenheimer: Zur Adsorptionskinetik an fluiden Phasengrenzen. Eine numeriache Lösung für den diffusionskontrollierten Adsorptionsvorgang. Mit 4 Abb. (Eing. 14. 2.77).

R. Mrtzner und C.-R. Kramer: Zur basischen Hydrolyse von Phenoxyessigsäureestem. III. Zur basischen Hydrolyse von 2,4-Dichlorphenoxyessigsäurealkylestern. 2. Teil. Mit $6 \mathrm{Abb}$. und 2 Tab. (Eing. 29. 3. 77)

F. MAtA and A. Mucientes: Kinetic Study of the Hydrolysis of Ortho and Para Methyl Hydroxibenzoates. With 2 Fig. and $6 \mathrm{Tab}$. (Received 11.11.76, in rev, version 18. 2. 77) ........

W. Schulz und W. GrüNDLER: Absolutberechnung der Adsorption eines Wasserstoffmoleküls an der (100)-Oberfläche von kristallinem Lithiumhydrid. Mit $3 \mathrm{Abb}$. und 1 Tab. (Eing. 26. 2. 77)

H. Kresse und Ch. SelbmanN: Dielektrische Untersuchungen an einem binären System aus zwei nematischen kristallin-flüssigen Substanzen. Mit 7 Abb. und 1 Tab. (Eing. 8. 2. 77, in rev.

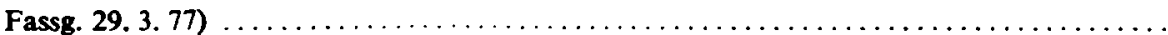

E. DoNTH: Fluktuationstheorie und Thermodynamik der Glasumwandlung in Polymeren. I. Dynamische Volumeneigenschaften und Temperatur-Zeit-Äquivalenz. (Eing. 14. 1. 77, in rev.

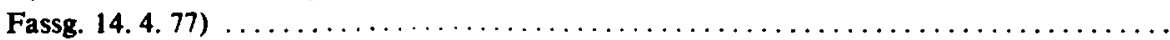

H. Stach, T. Peinze, K. FiEDler und W. SChirmer : Adsorptionswärme des n-Decans am Zeolith NaMgA. Mit 2 Abb. und 1 Tab. (Eing. 12.4.77)

D. Hartmann, G. Herzog und K. Schellmann: Luminophore auf der Basis aktivierter ZinkYttrium-Mischoxide für die Anwendung in Cadmiumdampf-Niederdruckentladungslampen. II. Emission und Anregung Bi-minoño-sowic Bi-Eu- und Bi-Dy-biaktivierter Zink-YttriumMischoxide unterschiedlicher Grundgitterzusammensetzung. Mit 7 Abb. (Eing. 26. 5. 77) ....

J. SAUer and Сн. Jung: Quantumchemical Perturbation Expansion for the Estimation of the Reaction Path in Radical-radical Reactions. With 4 Fig. and 2 Tab. (Received 10. 2. 77) ...

R. KümmeL und G. WILDE: Isopiestische Messungen im System Calciumnitrat-Harnstoff-Wasser

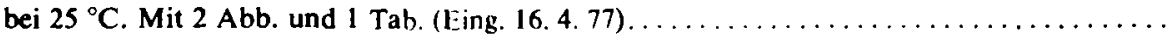

G. OPEL, U. STECHOW und E. VoGr L: Der Viskositätskoeffizient und der zweite Virialkoeffizient von Phenoldampf und ihre Darstellbarkeit durch Lennard-Jones- $(m-n)$-Ansätze für das intermolekulare Potential. Mit 3 Abh. und 5 Tab. (Eing. 25. 5. 77) ...................

G. RASCH, H. BöGEL und C. REIN: MO-Berechnungen an Koordinationsverbindungen. I. CNDORechnungen von Vanadin-Sauerstoff-Komplexen. Mit 5 Tab. (Eing. 24. 5. 77) . . . . . . .

J. SCHMELZER: Phasengleichgewichtsuntersuchungen an Mehrkomponentensystemen. III. Isobares Flūssigkeit-Dampf-Gleichgewicht und Flüssigkeit-Flüssigkeit-Gleichgewicht im quaternären System Methyläthylketon/sek. Butanol/Wasser/Diisobuten. Mit $4 \mathrm{Abb}$. und $2 \mathrm{Tab}$. (Eing. 12. 1. 77, in rev. Fassg. 6. 5.77)

U. Kroll und H. RöDicker: Zur Beschreibung des Löslichkeitsverhaltens von $\mathrm{C}_{3}$-Kohlenwasserstoffen in Hochdruck-Polyäthylen. Mit 4 Abb. und 4 Tab. (Eing. 23. 5. 77) ........

K. Fifdler, A. Roethe, K.-P. Roethe und D. Gelbin: Thermodynamische Konstanten in $\mathrm{NaMe}^{2+} \mathrm{A}-$ Zeolithen. I. Einfluß des Kations Me ${ }^{2+}$. Mit 5 Abb. und 3 Tab. (Eing. 13. 6. 77) . .

M. Borówko and M. JARONIEC: Competitive Adsorption of Binary Gas Mixtures on Homo-

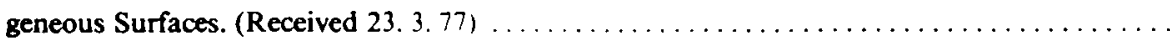

Kurze Mitteilungen

H. KeHLEN und E. Müller: Die Mischungswärmen von Butyläther-Butanol-Mischungen. Mit

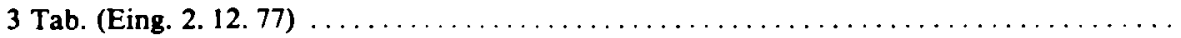

R. Khelawan and K. L. Yadava: Study of the Interaction of Hydrated Monopositive Iodine

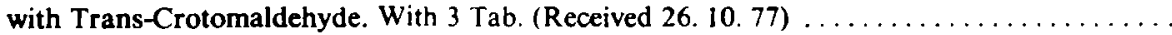

H.-H. Walter, H. Kaden und H. Hoffmaniv: Zur Bildung von Deckschichten auf Elektrodenoberflächen bei anodischer Polarisation in konzentrierten NaCl-Lösungen. Mit 3 Abb. (Eing. 29. 7. 76, in rev. Fassg. 16. 11. 77)

L. MülLER: Thermodynamische Begründung der Gültigkeit der linearen freien Enthalpiebeziehung für beliebige chemische Reaktionen. (Eing. 11. 1. 78) 
R. Ziebig, F. Pragst und W. Jugelt: Elektrochemisches Verhalten von N-Aryl- $\Delta^{2}$-pyrazolinen. XI. Elektrochemische Lumineszenz von 1,3,5-Triaryl- $\Delta^{2}$-pyrazolinen an der rotierenden Schei-

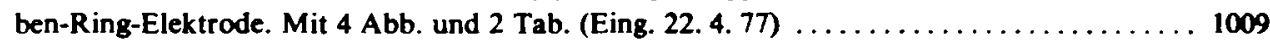

G. F. CerofolIni: Heterogeneity Effects in Desorption Kinetics. (Received 21. 2. 77) ...... 1020

D. Kumari Jha, K. P. ThakUR and B. N. Jha: Energetics and Mean Amplitude of Vibration of Alkaline Earth Chalcides and Heavy Metal Halides. With 3 Tab. (Received 8. 2. 77) ..... 1025

S. C. Sharma: Resonance Energies and Aromaticity of Some Benzenoids and Non-Benzenoids. With 2 Tab. (Received 17. 11. 76, in rev. version 15.4. 77) ................. 1031

H. Schulze und G. SchumanN: Zur Massentransportbestimmung der Diffusionskoeffizienten in nematischem MBBA. (Eing. 17.6. 77) ...

E. Donth: Fluktuationstheorie und Thermodynamik der Glasumwandlung in Polymeren. II. Einfrierproze $B$ und Glaszustand. Der Zusammenhang zwischen thermischer und dynamischer Glasumwandlung. Mit 1 Abb. (Eing. 14. 1. 77, in rev. Fassg. 14. 4. 77) $\ldots \ldots \ldots \ldots \ldots \ldots$

L. MülLER: Die Bestimmung der kinetischen Ströme bei der Uberlagerung von elektrochemischer Durchtrittsreaktion und Diffusion im Bereich kleiner Uberspannungen $(\eta<100 \mathrm{mV})$. Mit 1 Abb. und 1 Tab. (Eing. 7. 6. 77)

H. Wiechert, H. KRIENke, R. Feistel und W. EbeLING: Interionische Wechselwirkungen und Thermodynamik starker Elektrolyte. II. Alkalihalogenide bei $25^{\circ} \mathrm{C}$ in $\mathrm{H}_{2} \mathrm{O}$. Mit $11 \mathrm{Abb}$. und

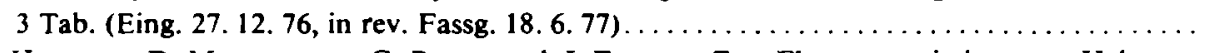

G. Henrion, D. MARquardt, G. Rietz und I. Fabian: Zur Flammenemission von Halogenkohlenstoffverbindungen. Mit 5 Abb. und 1 Tab. (Eing. 13. 7. 77)

M. S. A. Abd El-Mottaleb, A. M. El-Arrash and M. M. Abu-Ali: Spectrophotometric Studies and Hueckel Molecular Orbital Treatment of the Molecular Complexes of Iodine with Some Thiourea Derivatives. With 2 Fig. and 4 Tab. (Received 5. 5. 77) $\ldots \ldots \ldots \ldots \ldots \ldots \ldots$

J. OpfermanN und H.-H. Hörhold: Untersuchungen über Poly(arylenvinylen)e. 19. Untersuchung der spektralen Verteilung der Radikalionengeneration und deren Abhängigkeit von der

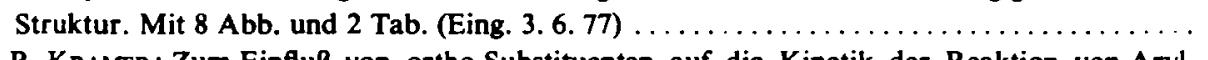

C.-R. Kramer: Zum EinfluB von ortho-Substituenten auf die Kinetik der Reaktion von Arylcarbonsäurehydraziden mit Bromcyan. Mit 5 Abb. und 2 Tab. (Eing. 24. 5. 77) ..........

I. Nagata, Y. Kawamura, H. Asano, K. Fujiwara and Y. Ogasawara: Excess Enthalpies for Binary Mixtures of Chloroform with Alcohols. With 1 Fig. and 2 Tab. (Received 20. 6. 77)... 1109

R. Namasivayam and K. Viswanathan NaIR: Molecular Constants of NSCl Molecule. With

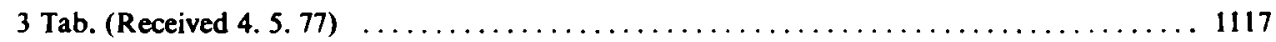

A. Lachowicz, A. Tröber, I. Bieräugel und B. AdLer: Untersuchungen an Vanadinsäureestern. III. Energetische Daten von Trialkylorthovanadaten. Mit 2 Abb. und 5 Tab. (Eing. 31. 5. 77, in rev. Fassg. 20.7.77).

G. A. Kokovin und T. ThIEME: Zur Thermodynamik des Systems Silizium-Jod-Wasserstoff. Mit 5 Tab. (Eing. 5. 8. 77)

W. Oehme, D. Freude, S. Klepel, H. Pfeifer und H. Schmiedel: Untersuchung von Hydtoxylgruppen hydratisierter NaX- und NaY-Zeolithe mit Hilfe der magnetischen Kernresonanz.

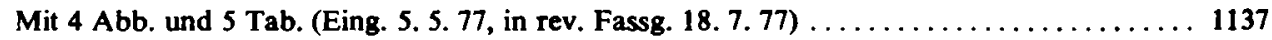

I. Nagata: Thermodynamic Properties of Alcohol-Inert Component Mixtures. With 2 Fig. and

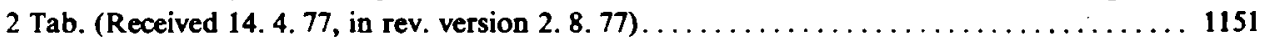

L. MũLleR: Zur Abhängigkeit der Geschwindigkeit elektrochemischer Reaktionen aus der Adsorptionsschicht von den Adsorptionsparametern Elektrode-Adsorbat. Mit 2 Tab. (Eing.

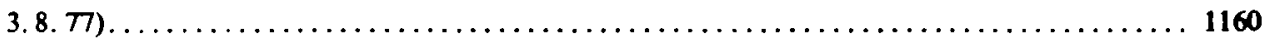


R. N. RaM and B. B. Prasad:: Study of the Catalytic Activity of Adsorbed Cobalt and Nickel Ions in the Decomprosittion of Hydrogen Peroxide. With 1 Fig. (Received 29.11. 76, in rev. version 5.8. 77) $\ldots \ldots \ldots \ldots \ldots \ldots \ldots \ldots \ldots \ldots \ldots \ldots \ldots \ldots \ldots \ldots \ldots \ldots \ldots \ldots \ldots .1169$

W. Lonserz und M. HalND elektroden. Mit 1 Tab. (IEing. 30.6.7-

Kurze Mitteilungen

C. Engler und W. LoreNz.: Liadungsverteilung aus lokalen Zustandsdichten an einem einfachen Chemisorptionsmodell. 'Mit' 2 Abb. (Eing. 30.6.77)

J. Srezkantha Babu anid K. Sirinfvastll: (hemical Oscillatory Systems - Gallic Acid and Bromate Reaction Catalyserd by Ferroln. With 3 Fig. and 1 Tab. (Received 9. 6. 77) . . . . 1191

Buchbesprechurgen . 1196

Anhang

Sachregister und Autoreraregister zM Band 258 (1977) 

